Exp. Anim. 55(2), 125-129, 2006

\title{
Ultrasonic Vocalization Response Elicited in Adjuvant-induced Arthritic Rats as a Useful Method for Evaluating Analgesic Drugs
}

\author{
Hiroyuki NAITO'), Takako OKUMURA ${ }^{3)}$, Maki INOUE ${ }^{1)}$, and Yoshihiko SUZUKI²)
}

\begin{abstract}
1)Department of Neurophysiology, 2)Department of Biochemistry, School of Veterinary Medicine, Azabu University, 1-17-71 Fuchinobe, Sagamihara 229-8501, ${ }^{3)}$ Discovery Biology Research, Nagoya Laboratories, Pfizer Global Research and Development, Pfizer Inc., 5-2 Taketoyo, Aichi 470-2393, Japan
\end{abstract}

\begin{abstract}
Adjuvant-induced arthritic (AIA) rats develop a severe chronic polyarthritis which shares some features in common with human rheumatoid arthritis. The purpose of the present study was to examine whether AIA rats emit ultrasonic vocalizations (USVs) when they are confronted with a healthy 'stimulus rat' in social interactions. We also examined the effects of three analgesic drugs (piroxicam, rofecoxib and ketoprofen) on USV responses using the same paradigm. In social interactions, AIA rats and intact controls emitted USVs in the $22-28 \mathrm{kHz}$ range. Vocalization activities were significantly higher in AIA rats than those in intact controls. Moreover, the USVs of AIA rats were significantly inhibited by the three analgesic drugs. These results suggest that the USV responses elicited in AIA rats are useful for the evaluation of analgesic drugs.
\end{abstract}

Key words: adjuvant-induced arthritic rats, ketoprofen, piroxicam, rofecoxib, ultrasonic vocalization

\section{Introduction}

Several animal models of chronic pain have been developed to evaluate pain mechanisms and the screening of analgesic drugs. Among the animal models of chronic pain, Freund's complete adjuvant-induced arthritis (AIA) in adult rats has been considered to be a useful animal model of chronic pain for the evaluation of analgesic drugs [1, 4-6], because the AIA rats' symptoms resemble the human condition with respect to inflammation and hyperalgesia. Evaluation of analgesic drugs has been accomplished by acute stimulation delivered to AIA rats. Pircio et al. [15], Okuyama and Aihara [14], Jourdan et al. [7], and Han et al. [9] reported that the audible and/or USV responses elicited in AIA rats by several acute stimulations were inhibited specifically by various analgesic drugs. Thus, validating that the audible and/or ultrasonic vocalization responses evoked in AIA rats might be an expression of pain.

Recently, Calvino et al. reported that ultrasonic vocalizations (USVs) in the $22-28 \mathrm{kHz}$ range with a $300-1200$ ms duration could also be elicited from AIA rats with chronic pain when they were confronted with

(Received 11 October 2005 / Accepted 23 January 2006)

Address corresponding: H. Naito, Department of Neurophysiology, School of Veterinary Medicine, Azabu University, 1-17-71 Fuchinobe, Sagamihara 229-8501, Japan 
a healthy 'stimulus rat' in social interactions [2]. Social interaction is known to elicit USVs from rats [10]. If the 'stimulus rat'-elicited USVs of AIA rats are actually a behavioral expression of chronic pain and can be inhibited by analgesic drugs, objective evaluation of the analgesic activity of newly developed drugs may become possible.

The purpose of the present study was to confirm whether USV responses can be elicited from AIA rats with chronic pain and to examine whether quantitative USV analysis is applicable to the assessment of analgesic drugs.

\section{Materials and Methods}

\section{Subjects}

We used 62 male LEW/CrlCrlj (Lewis) rats, 6 weeks of age, purchased from Charles River Japan, Inc. (Yokohama, Japan). Rats were housed in cages individually in an air-conditioned room $\left(22 \pm 2^{\circ} \mathrm{C}\right)$ with the lights on from $0700-2100 \mathrm{~h}$ and following the induction of adjuvant-induced arthritis, they were given free access to water and food directly available on the cage floor.

The induction of adjuvant-induced arthritis was performed by injection of $600 \mu \mathrm{g}$ of mycobacterium tuberculosis H37 RA (Difco Laboratories, Inc.) suspended in $100 \mu \mathrm{l}$ of liquid paraffin (Wako Pure Chemical Industries, Ltd.) into the right hindpaw. All of the injected animals showing macroscopic and behavioral signs of arthritis were tested 21 days after the injection. All procedures in this study were approved by the Animal Research Committee of Azabu University.

\section{Elicitation and measurement of ultrasonic vocalization}

AIA (56 rats) or intact subjects (6 rats) were placed in a neutral test cage alone for $10 \mathrm{~min}$, and USVs were recorded for $3 \mathrm{~min}$. Thereafter, social interaction with a 'stimulus rat' was tested. Five 8-month-old (430$460 \mathrm{~g}$ ) Tsukuba Low Emotional (TLE) male rats were used as the 'stimulus rat' for the elicitation of USV responses. They were chosen because they explore vigorously in a neutral test cage but rarely display aggressive behavior. For social interaction, a single 'stimulus rat' was put in a neutral test cage $(17 \times 21 \times$ $18 \mathrm{~cm}$ ) and an AIA or intact subject was presented for confrontation for at least $2 \mathrm{~min}$. USV responses were then recorded for $3 \mathrm{~min}$. If a rat did not emit USVs, another 'stimulus rat' was presented. Only those rats that responded and showed a USV response of longer than 3 min were used for assessing the effect of analgesic drugs.

The procedure for quantitative USV analysis has been described elsewhere [11-13]. In brief, ultrasonic waves transduced by a condenser microphone (Kunitachi Acoustic Lab., ACM-20F) installed $10 \mathrm{~cm}$ above the cage were amplified and fed to an electronic device (Diamedical System, DMP-350) measuring the average frequency and average amplitude of sine waves in 5-ms time bins which were successively output to a personal computer in real-time through an A/D converter. Sound production with frequencies higher than $20 \mathrm{kHz}$ was continuously monitored on a computer screen. For the waves with frequencies higher than $20 \mathrm{kHz}$, the average amplitude in a 5-ms time bin was accumulated for 3 min after starting measurement. The data are therefore combined products of the intensity and duration of USV. The data were expressed in arbitrary units.

\section{Assessment of effects of drugs}

'Stimulus rat'-elicited USV responses in AIA rats were measured for $3 \mathrm{~min}$ in the morning. Then, $2 \mathrm{~h}$ later, a non-steroidal anti-inflammatory drug was administered. USVs were then measured for $3 \mathrm{~min}$ beginning 30 and $60 \mathrm{~min}$ after the administration. The drugs used were piroxicam (Sigma) and ketoprofen (Capisten, Kissei Pharmaceutical Co.), which are cyclooxygenase (COX)-1 and COX-2 inhibitors, and rofecoxib (Vioxx, synthesized at Pfizer Gloval Research and Development), a selective COX-2 inhibitor. Piroxicam and rofecoxib were administered orally at a dose of $10 \mathrm{mg} / \mathrm{kg}$ body weight $(1.0 \mathrm{mg} / \mathrm{ml}$ suspension in $0.1 \%$ methylcellulose sol.). Ketoprofen was injected subcutaneously at a dose of $5.0 \mathrm{mg} / \mathrm{kg}$. Diazepam (Cercine, Takeda Pharmaceutical Co.) was used as an anxiolytic benzodiazepine receptor agonist and was injected subcutaneously at a dose of $1.0 \mathrm{mg} / \mathrm{kg}$ body weight $(0.5 \mathrm{mg} / \mathrm{ml}$ suspension in $0.9 \%$ saline with $1 \%$ Tween 80 ). Four or five days after each drug treatment, all rats were used once again in a test using the corresponding vehicles. The effects of the drugs and vehicles on the USV responses were expressed as the percentage of vocalization activity (VA\%) after administration relative to that before administration. 


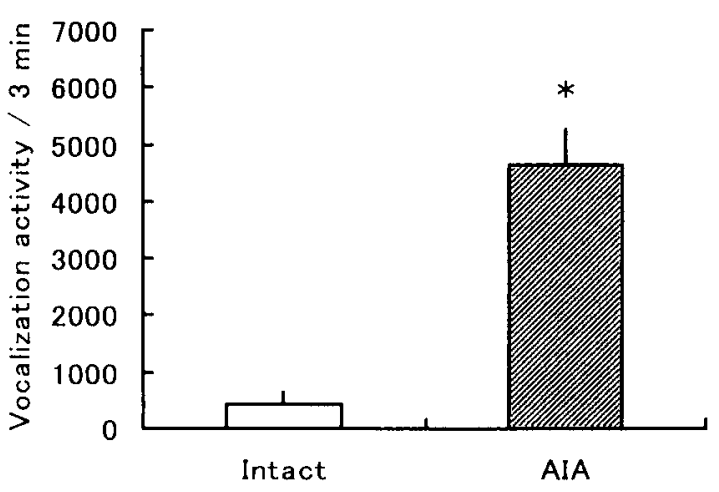

Fig. 1. Ultrasonic vocalizations of adjuvant-induced arthritic (AIA) rats $(n=12)$ and intact controls $(n=6)$ when confronted with single 'stimulus rats' in a social interaction. The asterisk denotes a significant difference $(P<0.05)$ from intact controls.

\section{Statistics}

The effects of drugs in the USV test were analysed by means of Student's $t$-test for comparison of means. All results are represented as the mean \pm SEM. A value of $P<0.05$ was considered significant.

\section{Results}

When the randomly selected AIA rats and intact controls were alone in the test cage, they scarcely exhibited USV responses. No statistical difference in vocalization activity was observed when compared with that of intact controls of the same strain $[0.36 \pm 0.12 / 3 \mathrm{~min}$ in AIA rats $(\mathrm{n}=6)$ vs $0.28 \pm 0.08 / 3 \mathrm{~min}$ in intact controls $(n=4), n s]$.

However, during social interaction in the neutral test cage, a single 'stimulus rat' was effective in eliciting a USV response in the $22-28 \mathrm{kHz}$ range with a $300-$ $1200 \mathrm{~ms}$ duration from the AIA rats $(\mathrm{n}=12)$ and intact controls $(n=6)$. AIA rats emitted 10 times more USVs than intact controls during this interaction, as shown in Fig. 1. A significant difference in vocalization activity was observed between AIA rats and intact controls $(P<0.05)$. USV response inducibility by the 'stimulus rat' was $92.86 \%(52 / 56)$ of the AIA rats. 'Stimulus rats' did not emit any USVs during this interaction.

The USV response of AIA rats was not attenuated by the injection of diazepam $(1 \mathrm{mg} / \mathrm{kg})$ when compared with those of vehicle-injected AIA rats $[30$ min: $\mathrm{t}=0.6606$, ns; 60 min: $\mathrm{t}=0.6525, \mathrm{~ns}]$, as shown in Table
Table 1. Effects of diazepam (1 mg/kg, s.c.) on the ultrasonic vocalization responses elicited from AIA rats $(\mathrm{n}=8)$ during social interaction

\begin{tabular}{lcc}
\hline \multirow{2}{*}{ Treatment } & \multicolumn{2}{c}{$\%$ of pretreatment } \\
\cline { 2 - 3 } & \multicolumn{1}{c}{$30 \mathrm{~min}$} & $60 \mathrm{~min}$ \\
\hline Vehicle & $100.35 \pm 4.85$ & $96.14 \pm 3.76$ \\
Diazepam & $96.23 \pm 3.60$ & $92.82 \pm 3.41$ \\
\hline
\end{tabular}

Vehicle or diazepam was given $30 \mathrm{~min}$ before the test. Data (mean \pm S.E.M.) of the vocalization activity after injection is expressed as a percentage of that before injection.

1. The effects of the three analgesic drugs on the USV response elicited from AIA rats are shown in Figs. 2-4. Piroxicam $(10 \mathrm{mg} / \mathrm{kg}$, p.o.) markedly inhibited the USV response. Significant differences were observed between AIA rats and vehicle-administered AIA rats at 30 and $60 \mathrm{~min}$ after the administration $[30 \mathrm{~min}$ : $\mathrm{t}=4.5606, P<0.01 ; 60$ min: $\mathrm{t}=22.6101, P<0.01]$. Ketoprofen $(5 \mathrm{mg} / \mathrm{kg}$, s.c.) markedly inhibited the USVs evoked in AIA rats at 30 and 60 min after the injection when compared with those of vehicle-injected AIA rats [30 min: $\mathrm{t}=7.3689, P<0.01 ; 60$ min: $\mathrm{t}=8.0241, P<0.01]$. Rofecoxib (10 mg/kg, p.o.) also reduced the USV responses significantly at 30 and $60 \mathrm{~min}$ after administration [30 min: $\mathrm{t}=2.1980, P<0.05$; $60 \mathrm{~min}$ : $\mathrm{t}=3.4266, P<0.01]$.

\section{Discussion}

The results obtained in this study confirm that AIA rats do not emit any USVs when they are alone in the test cage. However, AIA rats with chronic pain emitted ten times more USVs than intact controls of the same strain when confronted with a single 'stimulus rat' during social interactions (Fig. 1). Also, as shown in the vehicle-administered groups of Figs. 2-4, reproducibility of the USV response in the same animals was observed, if the 'stimulus rat' explored actively in the test cage.

The USVs in the $40-50 \mathrm{kHz}$ range associated with the threatening posture of the 'stimulus rat' were not measured during social interaction. The USVs in the $22-28 \mathrm{kHz}$ range recorded during social interaction were decreased significantly by the administration of analgesic drugs to AIA rats. We interpreted this as indicating 


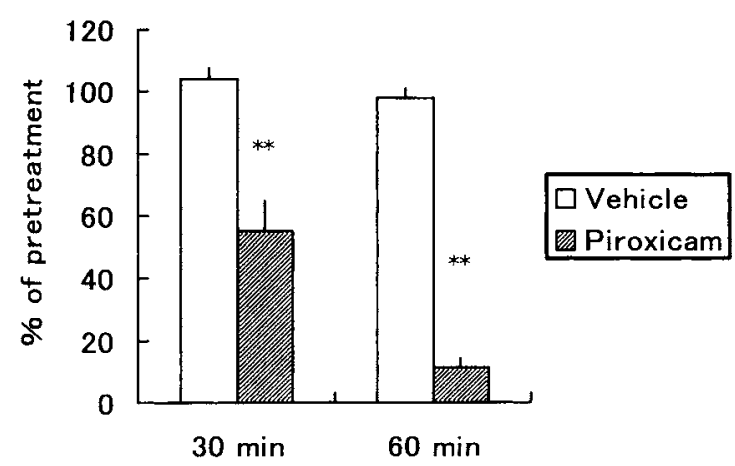

Fig. 2. Effect of piroxicam $(10 \mathrm{mg} / \mathrm{kg}$, p.o. $)$ on the ultrasonic vocalizations of AIA rats $(n=13)$ in social interaction. Data (mean \pm S.E.M.) of the vocalization activity after injection is expressed as a percentage of that before injection. The asterisks denote significant differences from vehicle-administered AIA controls: $* * P<0.01$.

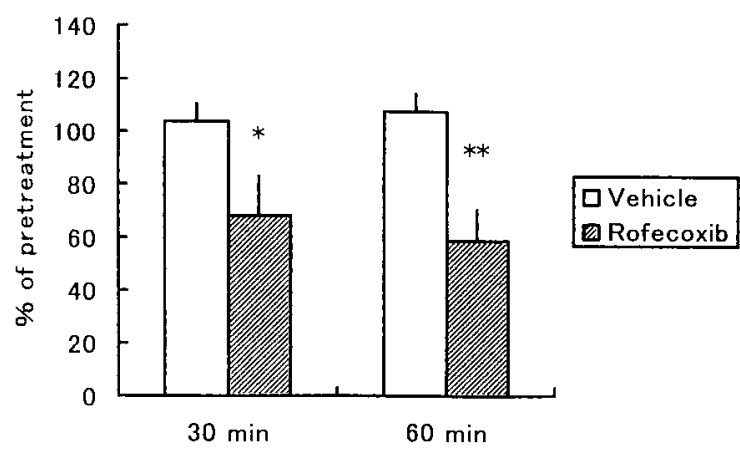

Fig. 3. Effect of rofecoxib (10 mg/kg, p.o.) on the ultrasonic vocalizations of AIA rats $(n=8)$ in social interaction. Data (mean \pm S.E.M.) of the vocalization activity after injection is expressed as a percentage of that before injection. The asterisks denote significant differences from vehicle-administered AIA controls: $* P<0.05, * * P<0.01$

that the 'stimulus rats' did not emit any USVs during the social interaction.

The USVs elicited from AIA rats were inhibited by all three of the non-steroidal anti-inflammatory drugs we tested, as shown in Figs. 2-4. The dosages used are known to have antinociceptive properties in conventional screening methods. However, the USVs evoked in AIA rats were not attenuated by diazepam $(1 \mathrm{mg} / \mathrm{kg}$, s.c.), an anxiolytic benzodiazepine receptor agonist, when compared with those of vehicle-injected controls. A train of pulses in the $22-28 \mathrm{kHz}$ range with a 300

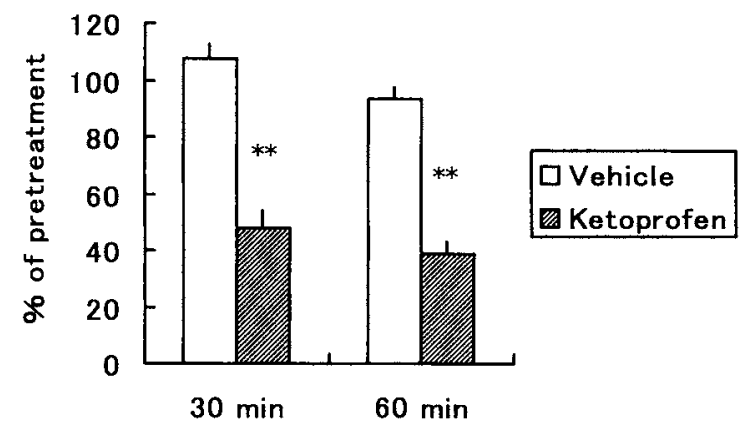

Fig. 4. Effect of ketoprofen $(5 \mathrm{mg} / \mathrm{kg}$, s.c.) on the ultrasonic vocalizations of AIA rats $(n=11)$ in social interaction. Data (mean \pm S.E.M.) of the vocalization activity after injection is expressed as a percentage of that before injection. The asterisks denote significant differences from vehicle-injected AIA controls: $* * P<0.01$.

1200 ms duration emitted by adult rats has been essentially associated with behaviors related to stressful and/ or painful situations, e.g. anxiety or stress, defense, and submission. Our results suggest that the USV responses of AIA rats during social interactions are associated with passive or submissive postures resulting from the chronic pain experienced by these subjects and are not the expression of a state of fear or anxiety. Calvino et $a l$. [3] reported that aspirin, a peripheral anti-inflammatory and analgesic compound with no anxiolytic properties, suppressed the USVs elicited from AIA rats during social interaction. This finding supports our results that the emission of USVs is not due to a state of anxiety of the AIA rats. Thus, a direct relationship between chronic pain and USV response in AIA rats is strongly suggested by the evidence. Therefore, quantitative analysis of the USV response as an indicator of chronic pain in AIA rats might be more advantageous for screening analgesic drugs than those of conventional screening methods using electrical, mechanical or thermal stimulations. The findings of Calvino et al. [3] agreed with the results of our present study. However, Jourdan et al. [8] reported that no difference in either the frequency or the duration of USVs was observed between healthy control rats and AIA rats during social interaction, and that aspirin had no effect on the USVs elicited from rats with chronic pain. Thus, they concluded that a direct relationship between USVs and chronic pain in AIA rats was not observed under their 
experimental conditions. Their result is not consistent with our present findings, and the cause of this discrepancy is unknown, because the same strain of rats was used in both experiments.

The defect of the 'stimulus rat' method for eliciting USV responses is that the vocalization activity of AIA rats is largely influenced by the locomotor activity of the 'stimulus rat'. When the locomotor activity of the 'stimulus rat' declines, the vocalization activity of the AIA rat also declines. To try to overcome this defect, plural 'stimulus rats' were used for confrontations at the same time for social interaction. This was not a decisive solution, however, so we are examining other methods to try and evoke a more consistent USV response in AIA rats.

In conclusion, AIA rats with chronic pain emitted USVs during social interactions. The USVs evoked in AIA rats were especially inhibited by the analgesic drugs we tested. Therefore, objective evaluation of analgesic activity may become possible through the quantitative analysis of USVs elicited from AIA rats.

\section{Acknowledgments}

We wish to thank Y. Fukuda for his skillful technical assistance.

\section{References}

1. Butler, S.H., Weil-Fugazza, J., Godefroy, F., and Besson, J.M. 1985. Reduction of arthritis and pain behaviour following chronic administration of amitriptyline or imipramine in rats with adjuvant-induced arthritis. Pain 23: $159-175$.

2. Calvino, B., Crepon-Bernard, M.O., and Le Bars, D. 1987. Parallel clinical and behavioural studies of adjuvant-induced arthritis in the rat: possible relationship with 'chronic pain'. Behav. Brain Res. 24: 11-29.

3. Calvino, B., Besson, J.M., Boehrer, A., and Depaulis, A. 1996. Ultrasonic vocalization $(22-28 \mathrm{kHz})$ in a model of chronic pain, the arthritic rat: effects of analgesic drugs. Neuroreport 7: 581-584.

4. Colpaert, F.C., Meert, T., De Witte, P., and Schmitt, P. 1982. Further evidence validating adjuvant arthritis as an experimental model of chronic pain in the rat. Life Sci. 31: $67-75$.

5. Colpaert, F.C. 1987. Evidence that adjuvant arthritis in the rat is associated with chronic pain. Pain 28: 201-222.

6. De Castro Costa, M., De Sutter, P., Gybels, J., and Van Hees, J. 1981. Adjuvant-induced arthritis in rats: a possible animal model of chronic pain. Pain 10: 173-185.

7. Jourdan, D., Ardid, D., Chapuy, E., Le Bars, D., and Eschalier, A. 1998. Effect of analgesics on audible and ultrasonic pain-induced vocalization in the rat. Life Sci. 63: 1761-1768.

8. Jourdan, D., Ardid, D., and Eschalier, A. 2002. Analysis of ultrasonic vocalization does not allow chronic pain to be evaluated in rats. Pain 95: 165-173.

9. Han, J.S., Bird, G.C., Li, W., Jones, J., and Neugebauer, V. 2005. Computerized analysis of audible and ultrasonic vocalizations of rats as a standardized measure of painrelated behavior. J. Neurosci. Methods 141: 261-269.

10. Haney, M. and Miczek, K.A. 1994. Ultrasounds emitted by female rats during agonistic interactions: effects of morphine and naltrexone. Psychopharmacology 114: 441448.

11. Naito, H., Nakamura, A., and Inoue, M. 1998. Ontogenetic changes in responsiveness to benzodiazepine receptor ligands on ultrasonic vocalizations in rat pups. Exp. Anim. 47: 89-96.

12. Naito, H., Inoue, M., Suzuki, Y., Tohei, A., Watanabe, G., Taya, K., and Makino, J. 2001. Ultrasonic vocalization response in genetically high- and low-emotional rats. Exp. Anim. 50: 285-291.

13. Naito, H., Nakamura, A., Inoue, M., and Suzuki, Y. 2003. Effect of anxiolytic drugs on air-puff-elicited ultrasonic vocalization in adult rats. Exp. Anim. 52: 409-414.

14. Okuyama, S. and Aihara, H. 1984. Inhibition of electricallyinduced vocalization in adjuvant arthritic rats as a novel method for evaluating analgesic drugs. Jpn. J. Pharmacol. 34: 67-77.

15. Pircio, A.W., Fedele, C.T., and Bierwagen, M.E. 1975. A new method for the evaluation of analgesic activity using adjuvant-induced arthritis in the rat. Eur. J. Pharmacol. 31: 207-215. 